\title{
Estrategia de responsabilidad social empresarial de una terminal portuaria en Barranquilla, Colombia*
}

\author{
Corporate social responsibility strategy of a harbour \\ terminal in Barranquilla, Colombia
}

\section{Estratégia de responsabilidade social empresarial de um terminal portuário em barranquilla, Colombia}

Recibido: 3 de agosto de 2018

Revisado: 31 de agosto de 2018 Aceptado: 30 de septiembre de 2018

\begin{abstract}
Jorge Isaac Lechuga-Cardo* $0^{* *}$
Universidad Autónoma de Nuevo León, México

Citar como: Lechuga-Cardozo, J. I. (2019). Estrategia de responsabilidad social empresarial de una terminal portuaria en Barranquilla, Colombia. SIGNOS - Investigación en sistemas de gestión, 11(1), 131-144. DOI: https://doi.org/10.15332/s2145-1389.2019.0001.08
\end{abstract}

\section{RESUMEN}

El presente artículo es producto de una investigación cuyo objetivo es caracterizar las actividades en responsabilidad social empresarial (RSE) que practica una terminal portuaria para posteriormente proponer acciones correctivas que ayuden al sostenimiento de este tipo de iniciativas. Se aplicó el método de análisis, con un enfoque cualitativo, de tipo descriptivo bajo un diseño documental, bibliográfico. Tras el análisis de los datos se encontró que la compañía goza de un marco ético basado en valores y principios corporativos alineados a su actividad económica, realizando acciones sociales desde cuatro pilares que responden a las

* Artículo de resultado de investigación.

* Candidato a doctor en Relaciones Internacionales, Negocios y Diplomacia, magíster en Administración de Organizaciones, especialista en Gerencia de Negocios Internacionales, administrador de empresas. Facultad de Ciencias Políticas y Relaciones Internacionales, Universidad Autónoma de Nuevo León, México. Correo electrónico: jorge.lechugacrd@uanl.edu.mx. ORCID: https://orcid.org/0000-0002-0999-5468 
dimensiones social, económica y ambiental; no obstante, se requiere fortalecer la instrumentación de medios interactivos y mediáticos. Como conclusión se sugiere a la terminal replicar los modelos de los departamentos de Bolívar y Sucre que los han hecho merecedores de distinciones, e integrar la inteligencia colectiva en su estrategia de responsabilidad social que le permita desarrollar en los empleados competencias como: la cooperación, el trabajo en equipo y el enfoque integrativo.

Palabras clave: responsabilidad social empresarial, inteligencia colectiva, terminal portuario.

\section{ABSTRACT}

The paper is the product of a research whose objective is to characterize the Corporate Social Responsibility (CSR) actions that a Harbour Terminal practices to later propose corrective actions that help sustain this type of initiatives. The method of analysis was applied, with a qualitative, descriptive approach under a documentary, bibliographic design. After the data analysis, it was found that the company enjoys of an ethical framework based on corporate values and principles aligned to its economic activity, developing social actions from four pillars that respond to the social, economic and environmental dimensions, nevertheless, it is required to reinvigorate the interactive media and mass media instrumentation. As a conclusion, it is suggested that the Terminal must replicate the models of the Bolivar and Sucre departments that have made it worthy of distinctions, and integrate collective intelligence into its social responsibility strategy that allows it to develop competences in employees such as: cooperation, teamwork and the integrative approach.

Keywords: Corporate Social Responsibility, collective intelligence, harbour terminal.

\section{RESUMO}

Este artigo é produto de uma pesquisa cujo objetivo é caracterizar as atividades em responsabilidade social empresarial (RSE) que pratica um terminal portuário para posteriormente propor ações corretivas que ajudem a conservação deste tipo de iniciativas. Aplicou-se o método de analise, com um enfoque qualitativo, de tipo descritivo sob um desenho documental, bibliográfico. Após a analise dos dados encontrou-se a que a companhia possui um marco ético baseado em valores e princípios corporativos alinhados com sua atividade econômica, realizando ações sociais desde quatro pilares que respondem às dimensões sociais, econômicas e ambientais; não obstante, se requer fortalecer a instrumentação de médios interativos e mediáticos. Como conclusão se sugere para 0 terminal replicar os modelos dos departamentos de Bolívar e Sucre que lhe tem merecido distinções, e integrar a inteligência coletiva em sua estratégia de responsabilidade social que permita desenvolver nos funcionários competências como: a cooperação, o trabalho em equipe e 0 enfoque integrador.

Palavras-chave: responsabilidade social empresarial, inteligência coletiva, terminal portuário.

\section{INTRODUCCIÓN}

En los últimos años la globalización de los mercados y los avances en la comunicación moderna vuelven a los habitantes de todo el mundo más sensibles ante una infinidad de temáticas cobrando especial importancia la responsabilidad social empresarial; de modo que existe un incremento de la exigencia social por un comportamiento empresarial más responsable y que va más allá de la maximización de ganancias (Aguilera y Puerto, 2012, p. 3).

Actualmente, este concepto se encuentra en la agenda empresarial de los diferentes sectores económicos en 
torno al rol que le compete a la empresa del siglo XXI (Pérez, Espinoza y Peralta, 2016, p. 170). Las terminales portuarias colombianas no están ajenas a esta nueva configuración y se comienza a percibir una tendencia hacia un desarrollo estructurado de este aspecto dentro de sus organizaciones.

El desafío de generar estrategias de responsabilidad social empresarial se impone ante el cumplimiento de normas y estándares internacionales que responden a las necesidades mucho más exigentes de consumidores globales (Camacho, 2015, p. 11). La mejor manera de abordar este desafío es conociendo prácticas exitosas de acciones de RSE, que permitan desarrollar estrategias efectivas de cooperación y de colaboración entre los diferentes actores sociales.

Entendiendo que la terminal portuaria no puede limitarse únicamente a ofrecer servicios, sino que debe incrementar sus objetivos considerando los intereses y necesidades de los colectivos que pueden verse afectados por las actividades de la empresa (Lechuga, Rodríguez y Alarcón, 2014, p. 160), más allá del cumplimiento normativo de parte de Compas (empresa objeto de estudio), debe existir una contribución voluntaria y activa para el mejoramiento de las dimensiones social, ambiental y económica.

El presente documento consiste en la elaboración de un estudio de caso que recoge el modelo de responsabilidad social empresarial utilizado en la terminal portuaria de Compas con sede en Barranquilla, Colombia, esto a través de un análisis de inteligencia colectiva.

El tema de investigación se desarrolla en el subsector logístico portuario (MinEducación, OEI y FITAC, 2017), donde confluyen y se interrelacionan de manera significativa el sector privado, la estructura del clúster portuario y la sociedad en general.

Las Naciones Unidas entiende la responsabilidad social corporativa (RSC) como el compromiso voluntario que las empresas hacen para la mejora social, económica y ambiental. Quiere ir más allá del cumplimiento de las leyes y las normas, dando por hecho su respeto y su estricto cumplimiento, que engloban un conjunto de prácticas, estrategias y sistemas de gestión empresariales, que persiguen un nuevo equilibrio entre las dimensiones económica, social y ambiental (ANUE, s. f.).

En esta investigación se identifica como problema la necesidad de la terminal portuaria por satisfacer las demandas, las necesidades y los intereses de sus grupos de interés (comunidad) a los que impacta con sus actividades. En este sentido, se plantea como pregunta problema: ¿¿cuáles son las acciones en responsabilidad social empresarial que practica una terminal portuaria de Barranquilla para la atención de la comunidad del barrio Las Flores como stakeholder?

Dentro de las motivaciones se encuentra conocer cuál es el modelo o enfoque ético, el soporte teórico y que sustente la intervención en la terminal portuaria, así como la definición de los instrumentos más adecuados para ese propósito.

En este sentido se genera conocimiento experto acerca de las acciones que una terminal portuaria en Barranquilla ejerce sobre su zona de influencia, en el barrio Las Flores, ubicado muy cerca de la desembocadura del río Magdalena, en el extremo noroccidental, habitado por pescadores artesanales, restauradores y trabajadores del puerto.

Como sugiere Carroll(1999), se requiere de herramientas que permitan una transformación social cimentada en valores comunitarios, generación de ingresos y empoderamiento cultural, que permitan construir una sociedad pacífica, justa y equitativa, contribuyendo al desarrollo social de la ciudad y al mejoramiento de la calidad de vida de sus habitantes.

En cuanto a la estructura del trabajo, esta se presentará de la siguiente manera: en primer lugar, se muestra la 
revisión de la literatura a través de los principales autores que soportan la investigación, seguidamente se expondrá la metodología utilizada en la investigación y los objetivos, luego se realiza un análisis de resultados, para finalmente desarrollar la discusión y conclusiones a la luz de los resultados obtenidos.

\section{REVISIÓN DE LITERATURA}

En una economía de mercado que exige cada vez más empresas competitivas que produzcan bienes y servicios que se adapten a las necesidades del entorno, se requiere de organizaciones con alta competitividad e innovación, pero esto no puede ser posible sin un marco ético (Lechuga, 2015, p. 132).

Pero, ¿a que atañe un marco ético dentro de la empresa? Sin lugar a dudas, a la ejecución de prácticas socialmente responsables, pues todas las organizaciones, públicas o privadas, tienen la obligación moral de la responsabilidad social. Empero, cuando se habla de responsabilidad social, se hace alusión a la adhesión voluntaria de las organizaciones a los factores ambientales y sociales en el día a día con la búsqueda del impacto en sus grupos de interés.

A continuación, se realiza una revisión del estado del arte de la responsabilidad social empresarial para la comprensión de su relación y el impacto que tiene en la sociedad la aplicabilidad de esta.

\section{Responsabilidad social empresarial}

En primer lugar, Aguilera y Puerto (2012) sostienen que la responsabilidad social empresarial actúa como un agente clave que impulsa el crecimiento de la empresa y favorece toda iniciativa que se origina en esta, facilitando el posicionamiento de su marca, renovando la imagen corporativa, capturando la preferencia y la lealtad de los clientes, así como mejora ostensiblemente la reputación y la credibilidad, y con ello logra el reconocimiento.

Complementan Andrade y Fernández (2010) que la responsabilidad social ha surgido en la sociedad en medio de madurez en la consciencia, debido a que ya no solo la empresa debe pensar en términos de los factores tradicionales, sino que debe atender a las exigencias de la dimensión ambiental, aspecto priorizado tanto por Gobiernos como por consumidores.

También, iniciativas como el Pacto Global de las Naciones Unidas, red de empresas e instituciones de todo el mundo en el que anualmente estas presentan su informe de progreso y demuestran su compromiso con los diez principios del Pacto Mundial de las Naciones Unidas.

Por otro lado, Cambra, Fuster, Polo y López (2010) identifican y analizan la importancia real de los factores que determinan el mayor o menor nivel de preocupación por el cliente y establecen relaciones entre los conceptos.

Agregan Navarro, Alcaraz y Ortiz (2010), que las prácticas de divulgación de la responsabilidad social están siendo ampliamente utilizadas por las grandes empresas como herramientas para eliminar asimetrías de información con sus grupos de interés. El estudio observa las administraciones locales españolas con el objeto de diagnosticarlas.

Del mismo modo, Avendaño (2013) expone que la responsabilidad social es la ética de la empresa con su entorno, relacionado con las decisiones de desarrollo, teniendo en cuenta las implicaciones para la empresa. Es decir, la empresa da respuesta a las expectativas e intereses de sus grupos de interés.

Por otro lado, Marquina y Refico (2015) realizan una investigación donde se analiza la relación entre 
responsabilidad social y habilidades empresariales para los consumidores en Bogotá, así como su disposición a pagar en presencia de estas variables en las empresas detrás de los productos. Encuentran que existen algunas iniciativas de responsabilidad social que tuvieron influencia significativa en las decisiones de consumo y decisión de compra de los consumidores. Por lo que se puede afirmar que el consumidor está cada vez más educado e informado por lo que exige hacer un consumo responsable en la compra de sus productos.

Agregan Barbosa, Medina y Vargas (2014) que la responsabilidad social se introduce como un mecanismo cuyo objeto es disminuir el deterioro medioambiental, la inequidad social, las crisis económicas y la corrupción en contexto globalizado, a pesar de que hoy son cuestionados su eficacia e impacto. En el artículo se identifican las tensiones estructurales que enfrenta el sistema económico y cómo las variables mencionadas aportan a la comprensión y solución de las dificultades.

No obstante, Méndez y Peralta (2014) manifiestan que la sociedad demanda de responsabilidad social sobre las empresas, pero estas últimas no asocian el concepto con creación de valor, ya que estas tienden a utilizarla como un instrumento de mercadeo develando sus buenas prácticas y ocultando las que tienen impacto negativo en sus stakeholders. Los autores realizan un análisis a partir de las relaciones económicas con la interdependencia social.

Calvo (2014) sostiene que la responsabilidad social se ha conceptuado desde diferentes miradas como filantropía, el mecenazgo, la estrategia o el marketing empresarial, en el estudio se centra la atención en cómo las empresas requieren bienes comunicativos como la reputación en la maximización de su beneficio económico, teniendo en cuenta la dimensión ética que la subyace.

Noe, Burachi y Peretti (2016) presentan una investigación que tiene por objeto determinar si los empresarios argentinos se orientan hacia la sostenibilidad o a la responsabilidad social empresarial, tomando como muestra 127 empresarios de la provincia de Córdoba en Argentina, concluyendo que los encuestados responden en su mayoría a un constructo de sostenibilidad y no al de responsabilidad social empresarial.

A partir de la revisión de los diferentes autores en materia de responsabilidad social empresarial, se puede asegurar que una empresa es socialmente responsable cuando independientemente de su tamaño tiene en cuenta los factores sociales, culturales y ambientales en la creación de su estrategia corporativa, para asegurar valor y ganancias. Es importante mencionar que esa estrategia debe ser creada a partir de la interacción con los grupos de interés, es decir, generándose procesos de inteligencia colectiva donde intervienen las comunidades, evidenciándose el conocimiento de los intereses y necesidades para desarrollar programas pertinentes que apunten a mitigar estas demandas sociales.

\section{Inteligencia colectiva 0 cointeligencia}

Hoy por hoy se proponen nuevas formas de inteligencia, donde el foco de estudio deja de ser el sujeto aislado para pasar a ser el grupo de sujetos, el colectivo o las multitudes. Así, numerosos autores contemporáneos han destacado el valor agregado de las multitudes en el desarrollo y construcción de conocimientos o incluso en la resolución de tareas (Atlee, 2003; Bloom, 2000; Rheingold, 2002; Surowiecki, 2005).

Levy, citado por Lobo y Pardo (2007), define a la inteligencia colectiva como la capacidad que tiene un grupo de personas de colaborar para decidir sobre su propio futuro, así como para alcanzar colectivamente sus metas en un contexto de alta complejidad (p. 147).

Se entiende entonces que cada persona sabe algo, por lo que nadie tiene el conocimiento absoluto, de manera que resulta importante la inclusión y participación 
del conocimiento de todos dentro del marco de la responsabilidad social empresarial de la compañía.

Por su parte, Levy (2004) señaló algunos dispositivos que permiten desarrollar la inteligencia colectiva (IC), a saber: 1) Los instrumentos de "vínculo social" encaminados a incluir al otro, a compartir y aprehender con él. 2) El aprovechamiento de los medios de comunicación masiva que buscan efectivamente "poner en común" las construcciones simbólicas de todos los hombres. 3) Los sistemas que persiguen como fin desarrollar en el hombre su autonomí. 4) El incremento de las "ingenierías semióticas" que posibiliten que los hombres puedan enriquecer sus competencias, acceder a los datos y apropiarse de la "potencia simbólica" de la humanidad (p. 76).

En particular, Bloom (2000) plantea la consideración de una CI grupal, argumentando que, si un test de CI individual compara habilidades individuales en la resolución de problemas con las capacidades de resolución de problemas de otros sujetos de la misma edad, de manera similar sería posible demostrar la existencia de inteligencia grupal comparando los desempeños de distintos grupos mientras resuelven problemas.

Según Atlee (2003), la definición de cointeligencia es la capacidad que se extiende más allá del coeficiente intelectual individual y que involucra distintos fenómenos relacionados con la inteligencia puesta en acto como resultado de la participación de más de un individuo.

Surowiecki (2005) argumenta que "[...] dado un conjunto de circunstancias adecuadas, los grupos manifiestan una inteligencia notable, y con frecuencia son más listos que los más listos de sus miembros" (p. 13).

Toca (2014) sugiere posibilidades de aplicación de la inteligencia colectiva no solo en especies sino en niveles de vida superiores como comunidades y ecosistemas. Concluye que en el largo plazo la flexibilidad y la robustez emergen de la autoorganización, se sugiere el estudio de los asuntos de esta última característica en redes empresariales (información, comunicación, liderazgo, potencial creativo, pertenencia, autonomía, acción colectiva, cooperación, interacción, libertad y diversidad), así como el análisis de redes soportado en grafos e indicadores.

\section{METODOLOGÍA}

El enfoque de la presente investigación es cualitativo, ya que las técnicas que se utilizarán para la recolección de datos, como la revisión documental, ayudarán a recopilar información y como bien lo dicen Taylor y Bogdan (1986), "es aquella que produce datos descriptivos: las propias palabras de las personas, habladas o escritas, y la conducta observable".

El alcance establecido es el descriptivo, puesto que pretende especificar propiedades, características y rasgos importantes de cualquier fenómeno que se analice. De igual forma, con este estudio descriptivo se busca especificar las propiedades, procesos, objetos o cualquier otro fenómeno que se someta a análisis (Hernández, Fernández y Baptista, 2014, p. 92). Es decir, únicamente pretende medir o recoger información de manera independiente o conjunta sobre los conceptos o las variables a las que se refieren, esto es su objetivo.

El diseño de investigación escogido es el transversal, porque el estudio que se busca hacer no tendrá un seguimiento en el tiempo y se efectuará en un período determinado (Hernández, Fernández y Baptista, 2014, p. 154).

\section{Instrumentos}

Para la construcción del marco teórico-conceptual de la estrategia de responsabilidad social de una terminal portuaria en Barranquilla, se acude a la revisión 
documental de fuentes primarias y secundarias de carácter empresarial y científico. Asimismo, se consultaron un total de 28 referencias bibliográficas utilizándose como instrumentos las ideas, argumentos y proyectos que fueron interpretados desde una perspectiva analítica y crítica.

\section{Procedimiento}

Con relación a la comprensión del problema de la investigación, se recopilan fuentes secundarias de documentos académicos. En el marco de referencia se definen los conceptos básicos relativos al marco ético de la teoría de la responsabilidad social empresarial. Una vez recopilada y analizada la información se construye el documento objeto de este trabajo. Por último, se realizan las recomendaciones y conclusiones conforme a los objetivos trazados.

\section{RESULTADOS Y DISCUSIÓN}

\section{Ubicación geográfica 0 espacio-temporal}

Empresa nacional con domicilio en Bogotá, terminales en Buenaventura, Barranquilla, Santiago de Tolú, Cartagena, Houston (EE. UU.) y Colón (Panamá). Su espacio virtual se encuentra en la página web oficial: http://www.compas.com.co/es/

Como compañía de puertos multipropósito, está en capacidad de prestar los servicios logísticos que la carga requiera. Entre otras, atiende el cargue y descargue de buques portacontenedores, buques RORO, buques graneleros, buques especiales para cargas de proyectos y cualquier artefacto naval que necesite servicios de estiba o desestiba de su carga.

Su portafolio de servicios para empresas importadoras y exportadoras incluye el llenado simultáneo de camión a contenedor o viceversa, la descarga de buques o camiones y el almacenamiento de las cargas en bodegas o silos especializados. Adicionalmente, maneja diversos tipos de cargas que necesitan equipos especiales como bobinas de papel, sacos y big bags ${ }^{1}$ en diferentes presentaciones y, en general, atiende todas las necesidades que la carga requiera.

A las navieras les ofrece ser su HUB de contenedores y carga suelta de transbordo internacional y a la industria nacional la posibilidad de manejar cargas bajo el régimen de depósito público de apoyo logístico internacional (Compas, s. f.).

\section{Cuerpo ético que implementa}

La estrategia de RSC y sostenibilidad del terminal portuario consiste en trabajar en línea con valores que los acercan y permiten crear relaciones de valor con todos sus grupos de interés. "Estos valores orientan su desarrollo como organización, demarcan una ruta de conducta para cada uno de los colaboradores que conforman la compañía y guían sus acciones para la consecución de los objetivos corporativos" (Compas, s. f.). El sistema de valores de Compas está integrado principalmente por los siguientes principios: integridad, respeto, trabajo de equipo, agilidad, innovación y calidad.

\section{Principios básicos de gestión organizacional}

Los principios que orientan el desarrollo de la organización demarcan la ruta para sus colaboradores y guían las acciones de los mismos hacia el alcance de los objetivos de la compañía, a saber son:

Seguridad: la seguridad es fundamental en su operación. Cada una de los terminales cuenta con los más avanzados sistemas de vigilancia, circuitos cerrados de

1 También conocidos como bolsones de polipropileno, contenedores flexibles a granel. 
televisión y equipos de seguridad. Gracias a esto, el esquema de protección de la terminal de Cartagena fue avalado por la aduana de Estados Unidos, como el prototipo para el desarrollo del estándar de seguridad BASC a nivel mundial.

La terminal portuaria también pertenece al programa de seguridad portuaria, integrado por la Fiscalía General de la Nación, la Policía Antinarcóticos y la Embajada de Estados Unidos, y cuenta con el apoyo de la Embajada de Francia.

La terminal portuaria ha sido reconocida en cuatro ocasiones con el Premio del Consejo Colombiano de Seguridad a la Gestión en Salud Ocupacional (2003, 2006, 2008 y 2010) y con el Premio Excelencia SURATEP en el año 2004.

Es la primera organización elegida como prototipo de seguridad en Latinoamérica por el Business Alliance for Secure Commerce (Alianza para el Comercio Seguro), programa liderado por la aduana norteamericana para bloquear el envío de drogas a través de embarques comerciales (Compas, s. f.).

Sostenibilidad: la terminal portuaria está comprometida con proyectos de sostenibilidad que involucran y benefician a todos sus grupos de interés, dentro de las cuales realizan actividades como: 1) Estrategia de agricultura sostenible: en los departamentos de Bolívar y Sucre, liderada por la Fundación Promotora del Canal del Dique del Puerto, que busca promover el cultivo de yuca biofortificada ${ }^{2}$, para lograr la seguridad alimentaria y el desarrollo rural de los agricultores. Iniciativa premiada con el Premio Marítimo de las Américas 2015, en la categoría Responsabilidad Empresarial en Puertos. 2) Proveedores sostenibles: acompañamiento a proveedores, contratistas y operadores portuarios para buscar la sostenibilidad de sus negocios (Compas, s. f.).

2 Es la idea de mejorar cultivos.
Relaciones de confianza: "La empresa cuenta con personas especializadas en la movilización de diversas cargas como hidrocarburos, contenedores, carbón, fertilizantes, vehículos y graneles, entre otras. La experiencia y preparación de su equipo humano es un importante activo" (Compas, s. f.).

Formación: "Mejora la empleabilidad en las comunidades donde operan, a través de la formación educativa" (Compas, s. f.).

Derechos humanos: la empresa apoya y respeta la protección de los derechos humanos fundamentales reconocidos universalmente, dentro de su ámbito de influencia. La empresa se asegura de no ser cómplice de la vulneración de los derechos humanos.

Responsabilidad ambiental y social: la terminal portuaria "fomenta este principio, capacitando a empleados, comunidad y aliados. En el año 2019 la terminal portuaria será líder reconocido en sostenibilidad y en la prestación de servicios logísticos integrados al comercio exterior" (Compas, s. f). Continuamente la empresa lleva a cabo programas que permiten aportar a la conservación del medio ambiente en general.

Desarrollo empresarial: "se proyectan inversiones a 2019, superiores a \$ 414 millones de dólares en la adecuación, modernización y especialización de nuestras terminales, que permitirán alcanzar una capacidad anual de movilización superior a 20 millones de toneladas de carga" (Compas, s. f.).

Objetos-enlace, ingeniería de vínculo, categorías de análisis

En la terminal portuaria el objeto es el conocimiento llamado intelecto colectivo, el enlace son los grupos de interés o stakeholders, se definieron como aquellos grupos e individuos que son, pueden o creen ser afectados por algún aspecto de las actividades de la empresa y 
por aquellos que tienen o pueden tener algún interés 0 pueden afectar a la organización. Son ellos: los socios, los proveedores de bienes y servicios, los colaboradores, la comunidad y los clientes. Las herramientas utilizadas para generar los espacios de conocimiento en el puerto son: análisis PESTAL, benchmarking, Qlikview³, análisis DOFA, meta mega y SET de mejores prácticas.

\section{Instrumentos mediáticos e interactivos que implementan}

La terminal portuaria interactúa con sus públicos a través de su página web, actualmente no hace presencia en redes sociales como Twitter, Facebook, LinkedIn y YouTube. Las comunicaciones con los clientes hoy por hoy se están realizando vía correo electrónico a través de la base de datos de los clientes y se utiliza la herramienta Microsoft Office 365 para establecer contacto con los colaboradores y proveedores. En la página web ofrecen servicios, terminales, contacto, sostenibilidad y sala de prensa.

\section{Temas centrales en los que intervienen}

La terminal portuaria contribuye con sus recursos a la mejora social y medioambiental del planeta a través de proyectos dirigidos al uso eficiente del agua, materiales y energía (entre otros). Su responsabilidad social está enmarcada en cuatro líneas maestras:

Proveedores sostenibles: realizan acompañamiento a proveedores, contratistas y operadores portuarios para que logren la sostenibilidad en los negocios (Compas, s. f.).

Agricultura sostenible: la terminal portuaria busca promover el cultivo de yuca, batata y frijol caupi biofortificados, para lograr la seguridad alimentaria y el

3 Herramienta de inteligencia de negocios que permite medir y realizar el seguimiento de procesos clave dentro de la empresa. desarrollo rural de los agricultores a partir de tecnologías avanzadas en el manejo ecoeficiente del suelo y el cultivo. A la fecha se han beneficiado 35 productores en Mahates, Bolívar. La siguiente etapa contempla la réplica del programa con 200 familias en la vereda El Palmar en el municipio de Tolú, Sucre (Compas, s. f.).

Voluntariado corporativo: los colaboradores de la terminal donan parte de su tiempo para actividades como elaboración de herramientas didácticas para niños, niñas y jóvenes de instituciones educativas, así como la siembra de huertas ecológicas (Compas, s. f.).

Negocios inclusivos: la compañía entregó dos quioscos en los terminales de Tolú y Barranquilla, que son administrados por la comunidad y se proyectan como espacios de ventas de refrigerios para empleados y contratistas. Además se incluyen capacitaciones, como asesorías administrativas y técnicas (Compas, s. f.).

\section{Aplicación de modelos teóricos 0 propuestas alternativas}

El terminal portuario promueve las inteligencias relacional y emocional a través de diferentes iniciativas sociales dirigidas a todo público acompañadas de la sensibilidad social de la compañía:

Entorno económico: garantiza la continuidad operativa del negocio, invierte en la modernización operativa de sus terminales, son eficientes y eficaces en la gestión de los recursos para mejorar la rentabilidad (Compas, s. f.).

Entono ambiental: la terminal controla y hace seguimiento de la disposición de los residuos y controla las emisiones que puedan contaminar el aire, el suelo y el agua (Compas, s. f.).

Gestión social: la terminal mejora la empleabilidad en las comunidades donde opera, articulando programas de formación con diversas organizaciones para 
desarrollar y fortalecer sus habilidades técnicas, en las que se enmarcan programas por la educación escolar y la protección del recurso hídrico y se establecen acuerdos con las comunidades para trabajar proyectos de inclusión social, educación y empleabilidad. La terminal educa y sensibiliza al personal interno a través de la realización periódica de voluntariado y mes de sostenibilidad (Compas, s. f.)

Tabla 1. Resultados de gestión social

\begin{tabular}{|l|c|}
\hline Comunidades de influencia directa & 15 \\
\hline Beneficiarios directos de la zona de influencia & 17.750 \\
\hline Beneficiarios a través de la Fundación Aguadulce & 6.104 \\
\hline Unidades de negocios comunitarios apoyados & 16 \\
\hline $\begin{array}{l}\text { Beneficiarios a través de la Fundación Canal del } \\
\text { Dique }\end{array}$ & 310 \\
\hline
\end{tabular}

Fuente: elaboración propia con datos de Compas, 2016.

\section{Plan estratégico de transición del modelo actual al modelo integral}

Una vez definidos los principios básicos de gestión organizacional con base en un cuerpo ético preciso, un marco teórico-práctico seleccionado como herramienta conceptual y la definición de las herramientas clave para ello, se presenta un ejercicio de asesoramiento o diseño de la estrategia de responsabilidad social del terminal portuario basado en principios de la inteligencia colectiva o cointeligencia.

A partir del plan estratégico actual se realiza la siguiente propuesta de plan estratégico con el objeto de realizar la transición del modelo operante al modelo basado en inteligencia competitiva o cointeligencia. 
Tabla 2. Plan estratégico

\begin{tabular}{|c|c|c|}
\hline Objetivo general & \multicolumn{2}{|c|}{$\begin{array}{l}\text { Caracterizar al terminal portuario como una empresa socialmente responsable que genera y promueve la } \\
\text { sostenibilidad dentro y fuera de la organización. }\end{array}$} \\
\hline Objetivo específico & Estrategia & Acciones \\
\hline $\begin{array}{l}\text { Enlazar la misión y la } \\
\text { visión de la organización } \\
\text { a los lineamientos } \\
\text { del modelo de } \\
\text { responsabilidad social } \\
\text { empresarial. }\end{array}$ & \multirow{5}{*}{$\begin{array}{l}\text { Desarrollar el talento } \\
\text { humano de la } \\
\text { organización mediante } \\
\text { la implementación } \\
\text { del coaching hacia las } \\
\text { competencias que } \\
\text { requiere la inteligencia } \\
\text { colectiva. }\end{array}$} & $\begin{array}{l}\text { Revisión de la misión y la visión. } \\
\text { Se hace necesario replantear la misión y la visión organizacional para que estas cumplan } \\
\text { con los lineamientos establecidos en el modelo de gestión de responsabilidad social, } \\
\text { se requiere realizar este ejercicio con la participación de todos los miembros de la } \\
\text { organización para garantizar que estas sean realmente interiorizadas. }\end{array}$ \\
\hline \multirow{4}{*}{$\begin{array}{l}\text { Adoptar como valores y } \\
\text { principios básicos de la } \\
\text { gestión de la organización } \\
\text { los que se identificaron } \\
\text { como coherentes con la } \\
\text { teoría de responsabilidad } \\
\text { social empresarial. }\end{array}$} & & $\begin{array}{l}\text { Determinar los valores y los principios } \\
\text { Teniendo en cuenta los propuestos por la asesoría, se deben definir mediante trabajo en } \\
\text { equipo los que la organización va a tomar e interpretarlos colectivamente. }\end{array}$ \\
\hline & & $\begin{array}{l}\text { Evaluación } \\
\text { Se requiere realizar una evaluación de } 360 \text { grados en todos los niveles de la organización } \\
\text { para identificar si los miembros de esta cuentan con las competencias acordes a los valores } \\
\text { y principios a establecer. }\end{array}$ \\
\hline & & $\begin{array}{l}\text { Desarrollo de competencias } \\
\text { Establecer un programa de desarrollo para todos los miembros de la organización de } \\
\text { acuerdo al resultado de la evaluación, este programa se realizará principalmente con } \\
\text { coachings externos e internos de acuerdo a las necesidades identificadas. }\end{array}$ \\
\hline & & $\begin{array}{l}\text { Sensibilización a través de la comunicación } \\
\text { Establecer un plan de comunicación que genere apropiación entre todos los miembros de } \\
\text { la organización de los nuevos principios y valores, principalmente utilizando la señalética. }\end{array}$ \\
\hline \multirow{6}{*}{$\begin{array}{l}\text { Generar sinergia en } \\
\text { los equipos de trabajo } \\
\text { mediante la gestión } \\
\text { y la utilización de las } \\
\text { herramientas mediáticas, } \\
\text { interactivas y virtuales } \\
\text { hacia la generación de } \\
\text { conocimiento colectivo. }\end{array}$} & \multirow{6}{*}{$\begin{array}{l}\text { Crear un sistema de } \\
\text { herramientas mediáticas, } \\
\text { interactivas y virtuales } \\
\text { mediante principios de } \\
\text { efectividad. }\end{array}$} & $\begin{array}{l}\text { Evaluar } \\
\text { Se requiere evaluar las herramientas que se están utilizando hasta el momento por la } \\
\text { empresa, determinar la utilización dentro y fuera de la empresa, el impacto logrado y si } \\
\text { realmente generan o no conocimiento colectivo. }\end{array}$ \\
\hline & & $\begin{array}{l}\text { Identificar } \\
\text { De las herramientas propuestas por la asesoría y de la evaluación anterior, determinar } \\
\text { cuáles se continúan utilizando y cuáles se requiere implementar para alcanzar el objetivo } \\
\text { propuesto. }\end{array}$ \\
\hline & & $\begin{array}{l}\text { Implementar } \\
\text { Adquirir y poner en marchas las herramientas identificadas. }\end{array}$ \\
\hline & & $\begin{array}{l}\text { Capacitar } \\
\text { Capacitar a todos los miembros de la organización en la utilización de las herramientas } \\
\text { establecidas y un grupo especial para gestionarlas. }\end{array}$ \\
\hline & & $\begin{array}{l}\text { Incentivar } \\
\text { Se puede unir al plan de comunicación establecido para el punto anterior la } \\
\text { sensibilización hacia la utilización y potencialización de estas herramientas, generar un } \\
\text { plan de incentivos ante la generación de sinergia de equipos de trabajo, de conocimiento } \\
\text { y otros. }\end{array}$ \\
\hline & & $\begin{array}{l}\text { Seguimiento } \\
\text { Determinar unos indicadores para medir la efectividad de estas herramientas, pero no } \\
\text { solo desde su utilización sino desde el verdadero impacto coherente con la inteligencia } \\
\text { colectiva. }\end{array}$ \\
\hline
\end{tabular}

Fuente: elaboración propia (2017). 
Como aseguran Cobo y Pardo (2007), en el contexto de la inteligencia colectiva se presenta un modelo integrador, donde el colectivo es el que produce diferenciación a la estrategia, teniendo como base la cooperación, el trabajo en equipo y el enfoque integrativo a través de estrategias que permitan a los grupos humanos generar sinergias con base en las herramientas que ofrece la Web 2.0, que suscitan la interacción y la creación de conocimiento.

En este sentido, se sugiere fortalecer los instrumentos interactivos y mediáticos del terminal portuario con sus grupos de interés, que le autoricen facilitar las relaciones y la comunicación con la organización, lo que permitirá nuevas dinámicas de construcción de capital social adoptando nuevos formatos de cooperación, interacción y coordinación; así como replicar las experiencias de los departamentos de Bolívar y Sucre en materia de negocios inclusivos y agricultura sostenible, con el objeto de que los programas ganen mayor visibilidad.

Se presenta como desafío importante seguir fomentando los indicadores de gestión social aumentando la cobertura de las iniciativas sociales a un mayor número de comunidades, unidades de negocios comunitarios y beneficiarios directos.

Por otro lado, una correcta integración de la estrategia de responsabilidad social en el ejercicio de planeación estratégica no solo le permitirá al terminal portuario desarrollar competencias en el talento humano de cointeligencia como la cooperación, el trabajo en equipo y el enfoque integrativo, sino ser un modelo referente de gestión social en el sector logístico portuario y también mejorar su reputación y proyección social.

\section{CONCLUSIONES}

A la luz de los objetivos planteados en el presente trabajo se presentan las siguientes conclusiones:
Como componentes centrales de la estrategia de responsabilidad social del terminal portuario en Barranquilla estudiado, se encontró que las acciones de responsabilidad social se encuentran alineadas a los valores corporativos, en los que se encuentran: integridad, respeto, trabajo de equipo, agilidad, innovación y calidad, teniendo en cuenta la actividad económica del terminal portuario que concentra operaciones logísticas portuarias en cargue y descargue de buques y almacenamiento.

El marco ético del terminal portuario esta sostenido sobre un aplicativo que incluye principios como la seguridad, las relaciones de confianza, la formación, los derechos humanos, la responsabilidad social-ambiental y el desarrollo empresarial; estos dan cuenta que los procesos están enmarcados en un compromiso por sus grupos de interés tanto internos como externos a través de acciones conscientes que conllevan a impacto positivo desde las dimensiones ambiental, económica y social.

Ahora bien, de acuerdo a Levy (2004) la transformación continua de las técnicas, de los mercados y del entorno económico lleva a las organizaciones a abandonar sus modos de organización rígidos y jerarquizados, a desarrollar las capacidades de iniciativa y de cooperación activa de sus miembros. Esta premisa se cumple cuando se evidencia en el terminal que existe una comunicación estrecha con sus públicos identificando y entendiendo sus demandas sociales.

Dentro de los instrumentos interactivos y mediáticos que implementa el terminal, se encuentran la página web y la intranet, desconociendo la oportunidad que tienen las redes sociales como medio de enlace con los públicos (Toca, 2014, p. 261).

Como postula Wilches (2011), las comunidades virtuales y redes sociales surgieron a principios del 2000, su popularidad demuestra que estamos tratando de 
establecer una relación simbiótica con la web, que no atañe al equipo que usamos todos a diario, sino que puede ser cualquier dispositivo que nos permite conectarnos a este sistema etéreo que nos rodea.

Su estrategia de responsabilidad social empresarial está centrada en líneas o componentes importantes como son: negocios inclusivos, voluntariado corporativo, proveedores y agricultura sostenible, donde realizan acompañamiento y brindan asistencia técnica en cada una de las líneas a los colectivos que integran a los empleados en función del servicio social a la comunidad.

Cabe destacar que esta estrategia le ha permitido ser merecedora de reconocimientos como el Premio Marítimo de las Américas 2015, otorgado por la Secretaría de la Comisión Interamericana de Puertos de la Organización de los Estados Americanos (OEA), en la categoría Responsabilidad Social Empresarial en Puertos, por su estrategia para la reducción de la pobreza en los departamentos de Bolívar y Sucre.

La estrategia de responsabilidad social actual está fundamentada en tres dimensiones: económica, ambiental y social, lo que incide positivamente en el desarrollo organizacional al no desvincular cada uno de los componentes sino por el contrario, integrarlos, esto le ha permitido a la compañía alcanzar indicadores de gestión social que le permiten materializar y visibilizar sus estándares en la acción de la responsabilidad social empresarial.

Finalmente, se propone al terminal fortalecer su estrategia de responsabilidad social empresarial desde la mirada de la inteligencia colectiva, comunicado sus acciones sociales a los públicos a través de las herramientas interactivas que proporciona la web, en especial de las redes sociales, movilizando y sensibilizando a los empleados hacia el compartimiento de los valores, e integrando la responsabilidad social a la plataforma estratégica actual de la empresa.

\section{REFERENCIAS}

Aguilera, A., y Puerto, D. (2012). Crecimiento empresarial basado en la responsabilidad social. Pensamiento \& Gestión, 32, 2-36.

Andrade, S., y Fernández, M. (2010). Responsabilidad social empresarial: significados e implicaciones a partir de la evolución de los procesos productivos. Producción Económica 2005, Recuperado de http://148.206.107.15/biblioteca_digital/ capitulos/375-5207icc.pdf

Asociación para las Naciones Unidas España - ANUE (s. f.). Responsabilidad social. Recuperado de http:// www.anue.org/es/content/responsabilidad-social

Atlee, T. (2003). Is collective intelligence like individual intelligence? The Co-Intelligence Institute. Recuperado de http://www.co-intelligence.org/CollectiveAndIndivIntell.html

Avendaño, W. (2013). Responsabilidad social (RS) y responsabilidad social corporativa (RSC): una nueva perspectiva para las empresas. Revista Lasallista de Investigación, 152-163.

Barbosa, D., Medina, C., y Vargas, M. (2014). Globalización, capitalismo financiero y responsabilidad social empresarial: tensiones estructurales. Civilizar, 14(27), 135-154.

Bloom, H. (2000). The global brain: The evolution of mass mind from the big bang to the 21st century. New York: N. Y. Wiley.

Calvo, P. (2014). Ética empresarial, responsabilidad social y bienes comunicativos. Tópicos, Revista de Filosofía, 47, 199-232.

Camacho, J. (2015). Las normas de responsabilidad social. Su dimensión en el ámbito laboral de las 
empresas. Revista Latinoamericana de Derecho Social, 20, 3-29

Cambra, J., Fuster, A., Polo, Y., y López, M. (2010). Preocupación por el cliente: ¿etica, responsabilidad o simplemente negocio? Innovar, 20(37), 47-58.

Carroll, A. J. (1999). Corporate social responsibility: Evolution of a definitional construct. Business and society, 38(3), 268-295.

Cobo, C., y Pardo, H. (2007). Planeta Web 2.0 inteligencia colectiva o medios fast food. México D. F., México: Flacso México.

Compas (s. f.). Portal oficial. Recuperado de http:// www.compas.com.co/es/

Hernández, R., Fernández, C., y Baptista, P. (2014). Metodología de la investigación. México D. F., México: McGraw-Hill.

Lechuga, J., Rodríguez, M., y Alarcón, C. (2014). Estado de conocimiento que tienen las instituciones de educación superior (IES) tecnológicas de Barranquilla y Cartagena sobre sus grupos de interés. Revista de Investigaciones UNAD, 13(2), 171-187.

Lechuga, J. (2015). Prospectiva estratégica, vigilancia tecnológica e inteligencia competitiva: el caso del Instituto Tecnológico de Soledad Atlántico de Colombia. Revista Academia \& Negocios, 1(2), 131-140.

Levy, P. (2004). Inteligencia colectiva: por una antropología del ciberespacio. Washington: La Découverte.

Marquina, P., y Refico, E. (2015). Impacto de la responsabilidad social empresarial en el comportamiento de compra y disposición a pagar de consumidores bogotanos. Estudios Gerenciales, 31(137), 373-382.

Méndez, J., y Peralta, D. (2014). Reflexiones respecto a la responsabilidad social empresarial y la creación de valor económico desde la perspectiva de los proveedores. Cuadernos de Contabilidad, 15(38), 625-645.

Ministerio de Educación de Colombia, Organización de Estados Iberoamericanos y Federación Colombiana de Agentes Logísticos en Comercio Internacional - FITAC, (2017). Marco Nacional de Cualificaciones Subsector Logística Portuaria. Bogotá D. C., Colombia: Eco Emprendedores Creativos S. A. S.

Navarro, A., Alcaraz, F., y Ortiz, D. (2010). La divulgación de información sobre responsabilidad corporativa en administraciones públicas: un estudio empírico en gobiernos locales. Revista de Contabilidad, 13(2), 285-314.

Noe, C., Burachi, M., y Peretti, F. (2016). Orientación de los empresarios de Córdoba, Argentina, hacia la sustentabilidad y la responsabilidad social empresarial: identificación de variables asociadas a cada constructo. Contaduría y Administración, 61(1), 84-105.

Pérez Espinoza, J. M., Espinoza Carrión, C., y Peralta Mocha, B. (2016).La responsabilidad social empresarial y su enfoque ambiental: una visión sostenible a futuro. Revista Universidad y Sociedad, 8(3), 169-178.

Rheingold, H. (2002). Smart Mobs: The Next Social Revolution. Nueva York: Basic Books.

Surowiecki, J. (2005). Cien mejor que uno. Barcelona, España: Editorial Urano.

Taylor, S. J., y Bogdan, R. (1986) Introducción: ir hacia la gente. En Introducción a los métodos cualitativos de investigación. México D. F., Mexico: Paidós.

Toca, C. (2014). Inteligencia colectiva: enfoque para el análisis de redes. Estudios Gerenciales, 30, 259-266.

Wilches, L. (2011). Módulo inteligencia colectiva. Bogotá D. C., Colombia: UNAD. 